\title{
High Grade Anal Canal Squamous Intraepithelial Neoplasia
}

National Cancer Institute

\section{Source}

National Cancer Institute. High Grade Anal Canal Squamous Intraepithelial Neoplasia. NCI

Thesaurus. Code C27275.

Intraepithelial neoplasia involving the anal canal. The epithelial cells exhibit moderate dysplasia (grade II intraepithelial neoplasia) or severe dysplasia (grade III intraepithelial neoplasia or carcinoma in situ). 